\title{
Crystal Structure of Pyridin-4-ylmethyl 4-Nitrobenzoate, $\mathrm{C}_{13} \mathrm{H}_{10} \mathrm{~N}_{2} \mathrm{O}_{4}$
}

\author{
James T. P. Matshwele, ${ }^{*}, *{ }^{*}$ Sebusi Odisitse, ${ }^{* \dagger}$ Ofentse Mazimba, ${ }^{*}$ Florence NAREetsile, ${ }^{* * *}$ \\ Lebogang G. JuLIUS, $* * *$ and Dalitso KeITUMETSE $* * *$ \\ *Department of Chemical and Forensic Science, Botswana International University of Science and Technology, \\ P/Bag 16 Palapye, Botswana \\ **Department of Applied Sciences, Botho University, PO Box 501564, Gaborone, Botswana \\ ***Department of Chemistry, University of Botswana, P/Bag UB704, Gaborone, Botswana
}

\begin{abstract}
The structure of pyridin-4-ylmethyl 4-nitrobenzoate was determined by the single-crystal X-ray diffraction method. The compound crystallized in a monoclinic system, $P 2_{1} / n$, and the unit cell parameters are, $a=9.981(2), b=12.347(3), c=$ 10.161(3) $\AA, \beta=101.450(9), Z=4, V=1227.3(5) \AA^{3}$. The crystal structure was solved by intrinsic phasing and refined to final values of $R 1=0.0451$ and $w R 2=0.1393$.
\end{abstract}

(Received August 21, 2021; Accepted August 31, 2021; Published on web January 10, 2022)

Pyridine derivatives have attracted much attention because of their medicinal properties, such as antifungal, antibacterial, antiinflammatory, antiviral, and antitumor activities. ${ }^{1-7}$ Furthermore, the benzoylation of these compounds may induce biological properties that makes it possible for ester group-containing compounds to act as prodrugs, and thus deliver the actual active group to the target biomolecule. ${ }^{8,9}$ The coordination of these types of ligands to certain metals has also shown enhanced medicinal properties. It was in this regard that influenced the synthesis of these types of pyridine derivatives. We wish to further coordinate them to metals and test for their bioactivity. However, this paper describes the crystal structure of an esterbridged pyridyl compound that has a nitro moiety bound at the para position.

In this research 4-nitrobenzoic acid $(2.80 \mathrm{~g}, 16.8 \mathrm{mmol})$ was mixed with $25 \mathrm{~mL}$ of dimethyl formamide. Potassium carbonate $(2.40 \mathrm{~g}, 17.4 \mathrm{mmol})$ was then added to the resulting mixture before refluxing, and the mixture was stirred for $30 \mathrm{~min}$ with a dry calcium chloride drying tube connected to the reaction vessel. Then, to the refluxed white precipitate obtained, 4-chloromethyl pyridine $(3.00 \mathrm{~g}, 18.3 \mathrm{mmol})$ dissolved in $25 \mathrm{~mL}$ dimethylformamide was added quickly before covering with a dry calcium chloride drying tube. The mixture was then refluxed overnight at $70^{\circ} \mathrm{C}$. Afterwards, the maroon precipitate obtained was cooled and neutralized with $10 \mathrm{~mL}$ of $10 \%$ sodium<smiles>O=C(OCc1ccncc1)c1ccc([N+](=O)[O-])cc1</smiles>

Fig. 1 Chemical diagram of the pyridin-4-ylmethyl 4-nitrobenzoate.

† To whom correspondence should be addressed. E-mail: odisitses@biust.ac.bw (S. O.); james.matshwele@ studentmail.biust.ac.bw (J. T. P. M.) hydrogen carbonate, $40 \mathrm{~mL}$ distilled water and filtered under suction. It was washed again with cold distilled water. Offwhite solid obtained was then air dried before characterization. The product was then recrystallized with methanol and golden rhombohedral crystals were obtained. Figure 1 shows the synthesized pyridin-4-ylmethyl 4-nitrobenzoate compound. The crystal and structure-refinement data are summarized in Table 1.

All hydrogen atoms were placed at geometrically calculated positions and included in the least-squares calculations using the riding-atom model. Their $U_{\text {iso }}$ values were fixed at $1.2 U_{\text {eq }}$ of

Table 1 Crystal data and structure refinement details

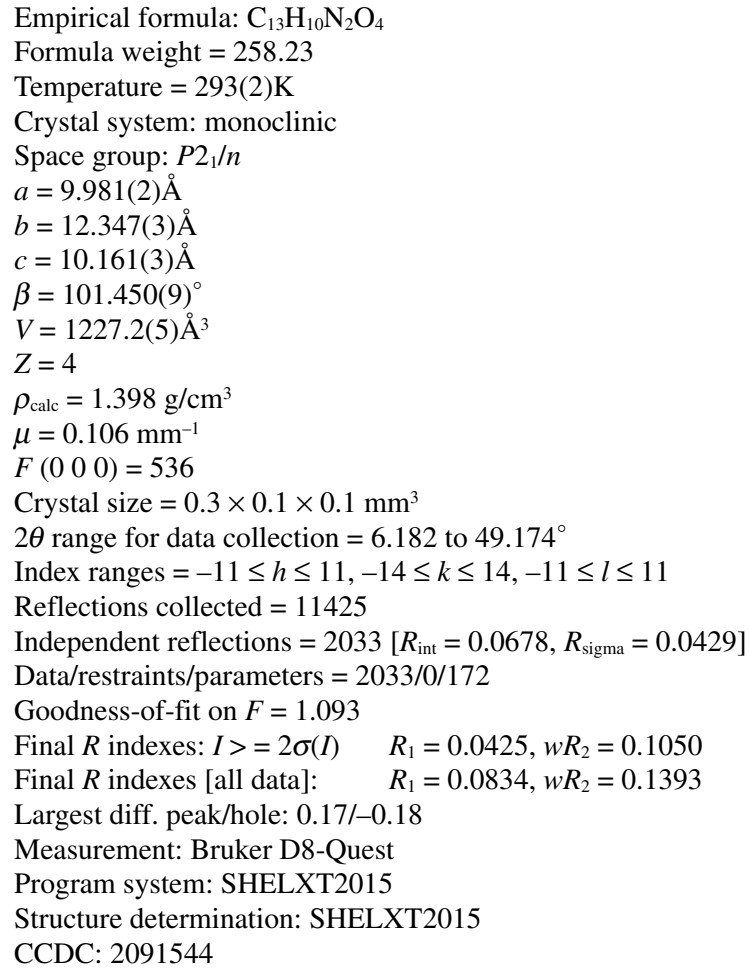




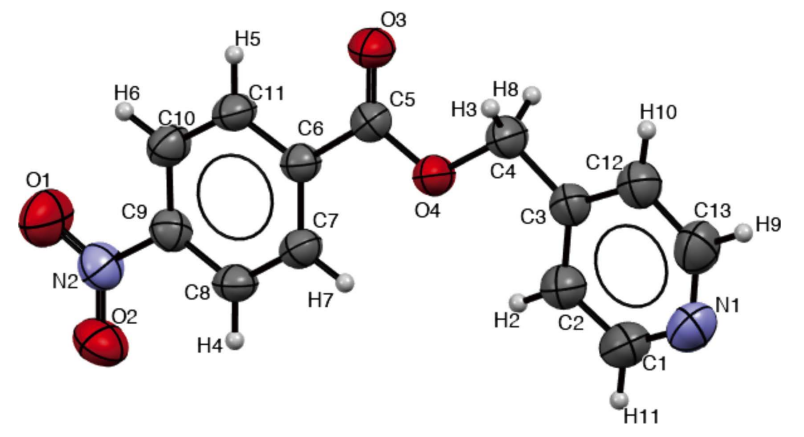

Fig. 2 ORTEP structure of pyridin-4-ylmethyl 4-nitrobenzoate, showing $50 \%$ probability ellipsoids.

Table 2 Selected bond angles

\begin{tabular}{lrlr}
\hline \multicolumn{1}{c}{ Bond } & Angle/ $^{\circ}$ & \multicolumn{1}{c}{ Bond } & Angle $^{\circ}$ \\
\hline C1-C2-C3-C4 & $-179.6(3)$ & C8-C9-N2-O1 & $-176.5(2)$ \\
C3-C4-O4-C5 & $177.8(2)$ & C10-C9-N2-O2 & $-176.8(2)$ \\
C4-C3-C12-C13 & $179.7(3)$ & C12-C3-C4-O4 & $-170.3(2)$ \\
C5-C6-C7-C8 & $-177.6(2)$ & N2-C9-C10-C11 & $180.0(2)$ \\
C5-C6-C11-C10 & $177.5(2)$ & O3-C5-C6-C7 & $-178.4(2)$ \\
C6-C5-O4-C4 & $175.0(2)$ & O4-C5-C6-C11 & $-176.4(2)$ \\
C7-C8-C9-N2 & $179.8(2)$ & & \\
\hline
\end{tabular}

their attaching carbon atoms.

The crystal structure of the reported molecule's analogue, 2-pyridyl 4-nitrobenzoate, has been reported before. ${ }^{10}$ The analogue was different in that it did not possess a methylene group that joins the ester to the pyridyl group. Our molecule was recovered as rhombohedral-shaped golden crystals. The crystal information of the molecules was similar. Also, the bond angles and lengths of the reported molecule were well within reported values and were similar to those of the analogue. They both crystallized in the monoclinic crystal system. The molecule transforms under the $P 2_{1} / n$ space group while the analogue also transformed under the $P 2_{1} / c$. As shown on the ORTEP structure on Fig. 2, the molecule's non hydrogen atoms and conjugated $\pi$ system exhibits planarity with a benzene ring substituted with a nitro group and an ester group on the para position. The torsion angles on Table 2 and especially the bonds C3-C4-O4-C5 177.8(2), C6-C5-O4-C4 175.0 (2), C7-C8-C9-N2 179.8 (2),
$\mathrm{C} 8-\mathrm{C} 9-\mathrm{N} 2-\mathrm{O} 1-176.5^{\circ}$ (2), C10-C9-N2-O2-176.8 ${ }^{\circ}$ (2), C12C3-C4-O4 -170.3 ${ }^{\circ}$ (2), N2-C9-C10-C11 $180.0^{\circ}$ (2), O3-C5-C6$\mathrm{C} 7-178.4^{\circ}$ (2) and O4-C5-C6-C11 -176.4 (2) define this planarity. Some inter-molecular contacts are observed on the expanded unit cell, we observed contacts of the type C-H6 $\cdots \mathrm{N} 1$ $2.530 \AA[x,-1+y, z], \mathrm{C}-\mathrm{H} 2 \cdots \mathrm{O} 22.553 \AA[1 / 2-x,-1 / 2+y, 1 / 2-z]$, $\mathrm{C}-\mathrm{H} 10 \cdots \mathrm{O} 32.563 \AA[2-x, 1-y, 1-z]$ and $\mathrm{C}-\mathrm{H} 4 \ldots \mathrm{O} 12.677 \AA$ $[1 / 2-x,-1 / 2+y, 1 / 2-z]$.

The CIF file is available as supporting material for all of the crystallographic information of the molecule. The file is freely available on the CSD database with the CCDC number 2091544.

\section{Acknowledgements}

The authors would like to give gratitude to the BIUST Office of Research, Development, and Innovation for the research grant S00326. Also, the University of Botswana for providing research facilities and the SC-XRD instrument.

\section{References}

1. Q. Li, L. A. Mitscher, and L. L. Shen, Med. Res. Rev., 2000, 20, 231.

2. Y. Fujita, H. Oguri, and H. Oikawa, Tetrahedron Lett., 2005, 46, 5885.

3. I. A Maghrabi, S. Alghamdi, M. Alrobaian, and H. A. Eldeab, Molecules, 2016, 21, 477.

4. A. Fassihi, D. Abedi, L. Saghaie, R. Sabet, H. Fazeli, G. Bostaki, O. Deilami, and H. Sadinpour, Eur. J. Med. Chem., 2009, 44, 2145.

5. P. Storck, A. M. Aubertin and D. S. Grierson, Tetrahedron Lett., 2009, 46, 2919.

6. G. Semple, B. M. Andersson, V. Chhajlani, J. Georgsson, M. J. Johansson, A. Rosenquist, and L. Swanson, Bioorg. Med. Chem. Lett., 2003, 13, 1141.

7. L. A Hasvold, W. Wang, S. I. Gwaltney, T. W. Rockway, L. T. Nelson, R. A. Mantei, S. A. Fakhoury, G. M. Sullivan, Q. Li, N. H. Lin, and L. Wang, Bioorg. Med. Chem. Lett., 2003, 13, 4001.

8. P. Praveen, S. Kabir, and T. Mohamed, Pharmaceuticals, 2010, 3, 1530.

9. J. M. Ferriz and J. Vinsova, Curr. Pharm. Design, 2010, 16, 2033.

10. M. L. Główka and I. Iwanicka, Acta. Crystallogr. Sect. C, 1990, 46, 1342. 